\title{
Kneser's theorems for strong, weak and pseudo-solutions of ordinary differential equations in Banach spaces
}

\author{
by Mieczysław Cichoń and Ireneusz Kubiaczyk (Poznań)
}

\begin{abstract}
We investigate the structure of the set of solutions of the Cauchy problem $x^{\prime}=f(t, x), x(0)=x_{0}$ in Banach spaces. If $f$ satisfies a compactness condition expressed in terms of measures of weak noncompactness, and $f$ is Pettis-integrable, then the set of pseudo-solutions of this problem is a continuum in $C_{\mathrm{w}}(I, E)$, the space of all continuous functions from $I$ to $E$ endowed with the weak topology. Under some additional assumptions these solutions are, in fact, weak solutions or strong Carathéodory solutions, so we also obtain Kneser-type theorems for these classes of solutions.
\end{abstract}

1. Introduction. We deal with the Cauchy problem

$$
\left\{\begin{array}{l}
x^{\prime}(t)=f(t, x(t)), \quad t \in[0, T] \\
x(0)=x_{0}
\end{array}\right.
$$

where $x^{\prime}$ denotes the weak derivative of $x$.

The study of the problem (1) was initiated by Szep [16], and theorems on the existence of weak solutions of this problem have been proved for example by Cramer, Lakshmikantham and Mitchell [5], Kubiaczyk and Szufla [12], Mitchell and Smith [13], Szufla [18] and Papageorgiou [14]. In all these papers the function $f$ is assumed to be weakly continuous, while in our paper we will only assume that $f$ is Pettis-integrable (for the definitions see [8], [10]). Our additional assumptions are expressed in terms of measures of weak noncompactness.

In the sequel, $(E,\|\cdot\|)$ will be a real Banach space. Then $E^{*}$ denotes the dual space, and $B_{r}=\left\{x \in E:\left\|x-x_{0}\right\| \leq r\right\}(r>0)$. Unless otherwise stated, we assume that ' denotes the weak derivative, and $\int$ denotes the Pettis integral. Denote by $I$ the interval $[0, T]$. Moreover, let $C_{\mathrm{w}}(I, E)$ denote the space of all weakly continuous functions from $I$ to $E$ endowed with the

1991 Mathematics Subject Classification: Primary 34A12, $47 \mathrm{H} 09$.

Key words and phrases: set of solutions, pseudo-solutions, measures of weak noncompactness, Pettis integral. 
topology of weak uniform convergence and let $(C(J, E), w)$ denote the space $C(J, E)$ with the weak topology $\sigma\left(C(J, E), C(J, E)^{*}\right)$.

Recall that a function $h: E \rightarrow E$ is said to be weakly-weakly sequentially continuous if $h$ takes each weakly convergent sequence in $E$ to a weakly convergent sequence in $E$.

In general, the space $E$ will be assumed to be nonreflexive (cf. Corollary 2).

2. Pseudo-solutions. Whenever we investigate the existence of strong solutions of (1) with discontinuous right-hand side, we consider so-called Carathéodory-type solutions [9] (strong $C$-solutions). For such solutions the problem (1) is equivalent to the integral problem

$$
x(t)=x_{0}+\int_{0}^{t} f(s, x(s)) d s, \quad t \in I .
$$

For the moment let ' denote the strong derivative, and $\int$ the Bochner integral. Recall that an absolutely continuous function $x: I \rightarrow E$ is said to be a Carathéodory-type solution of (1) if $x(0)=x_{0}$ and $x(\cdot)$ satisfies (1) a.e.

Fix $x^{*} \in E^{*}$, and consider

$$
\left(x^{*} x\right)^{\prime}(t)=x^{*} f(t, x(t)), \quad t \in I .
$$

First, we introduce the following definition:

Definition 1. A function $x: I \rightarrow E$ is said to be a pseudo-solution of the Cauchy problem (1) if it satisfies the following conditions:

(i) $x(\cdot)$ is absolutely continuous,

(ii) $x(0)=x_{0}$,

(iii) for each $x^{*} \in E^{*}$ there exists a negligible set $A\left(x^{*}\right)$ (i.e. $\operatorname{mes}\left(A\left(x^{*}\right)\right)$ $=0)$ such that for each $t \notin A\left(x^{*}\right)$,

$$
x^{*}\left(x^{\prime}(t)\right)=x^{*}(f(t, x(t))) .
$$

Here ' denotes the pseudo-derivative (see Pettis [15]).

In other words, by a pseudo-solution of (1) we will understand an absolutely continuous function such that $x(0)=x_{0}$, and for each $x^{*} \in E^{*}, x(\cdot)$ satisfies $\left(1^{\prime}\right)$ a.e.

We will deal with problem (2) with the Pettis integral. If $f$ is Pettisintegrable, then the existence of a pseudo-solution of (1) is equivalent to the existence of a solution of (2) (see [15], Section 8).

If we suppose the boundedness of $f$, then all pseudo-solutions are strongly absolutely continuous. Indeed, for fixed $s, t \in I$ there exists $x^{*} \in E^{*}$ with $\left\|x^{*}\right\|=1$ such that 


$$
\|x(t)-x(s)\|=x^{*}(x(t)-x(s)) \leq \int_{s}^{t}\left|x^{*}(f(\tau, x(\tau)))\right| d \tau \leq M|t-s| .
$$

There exist examples of problems of type (1) which only have pseudosolutions, but no weak solutions or strong $C$-solutions ([1], [4]).

3. Measures of weak noncompactness. The notion of a measure of weak noncompactness was introduced by De Blasi [7]. It can be used in the same way as the strong measure of noncompactness, for example in fixed point theory, and in the theory of functional equations or differential equations.

Let us denote by $\mathcal{N}_{\mathrm{w}}$ the family of all relatively weakly compact subsets of $E$, and by $\mathcal{M}$ the family of all bounded subsets of $E$. A function $\mu: \mathcal{M} \rightarrow$ $[0, \infty)$ is said to be a measure of weak noncompactness (cf. [3]) if

(i) $\mu(A)=0 \Leftrightarrow A \in \mathcal{N}_{\mathrm{w}}$,

(ii) $\mu(\overline{\operatorname{conv}} A)=\mu(A), A \in \mathcal{M}$,

(iii) $A, B \in \mathcal{M}, A \subset B \Rightarrow \mu(A) \leq \mu(B)$,

(iv) for each $x_{0} \in E, \mu\left(x_{0}+A\right)=\mu(A)$,

(v) $\mu(k A)=k A, k \in \mathbb{R}_{+}$.

LEMma 1 (cf. [2], [4], [13]). Let $H \subset C(I, E)$ be a family of strongly equicontinuous functions. Then the function $t \rightarrow v(t)=\mu(H(t))$ is continuous and $\mu(H(I))=\sup \{\mu(H(t)): t \in I\}$.

4. Main results. Now we prove a Kneser-type theorem for the problem (1). Let $f: I \times B_{r} \rightarrow E$ be such that $\|f(t, x)\| \leq M$ for each $(t, x) \in$ $I \times B_{r}$. Set $J=[0, d]$, where $d=\min \{T, r / M\}$.

THeORem 1. Assume that for each strongly absolutely continuous function $x: J \rightarrow E, f(\cdot, x(\cdot))$ is Pettis-integrable, $f(t, \cdot)$ is weakly-weakly sequentially continuous and

$$
\mu(f(J \times X)) \leq k \mu(X)
$$

for each bounded subset $X$ of $B_{r}$, where $k d<1$. Then the set $S$ of all pseudo-solutions of the Cauchy problem (1) on $J$ is nonempty, compact and connected in $(C(J, E), w)$.

Proof. Put $F(x)(t)=x_{0}+\int_{0}^{t} f(s, x(s)) d s$, where $\int$ denotes the Pettis integral. By our assumptions $F$ is a well defined operator from $\widetilde{B}=\{x \in$ $C(J, E): x(\cdot)$ is Lipschitz-continuous with constant $\left.M, x(J) \subset B_{r}\right\}$ into $\widetilde{B}$. Denote by $S$ the set of all fixed points of $F$ (i.e. $S=F(S)$ ). Equivalently, it is the set of all solutions of (1). 
Now, denote by $\Omega$ the family of all sets $W$ such that $S(J) \subset W \subset B_{r}$ and $R(W) \subset W$, where

$$
R(W)=x_{0}+\overline{\bigcup_{0 \leq \lambda \leq d} \lambda \operatorname{conv} f(J \times W)} .
$$

Then $B_{r} \in \Omega$. Indeed, $S(J) \subset B_{r}$, by assumptions on $f$ and since $\sup _{x \in A}\|x\|$ $=\sup _{x \in \overline{\operatorname{conv}} A}\|x\|$ and $R\left(B_{r}\right) \subset B_{r}$. If we denote by $H$ the intersection of this family, then $H \neq \emptyset$ since $x_{0} \in H$ (moreover, $\left.S(J) \subset H\right)$. For each $W \in \Omega$ we have $H \subset W$, so $R(H) \subset R(W) \subset W$ and consequently $R(H) \subset$ $\bigcap_{W \in \Omega} W=H$. Therefore $H \in \Omega$. Thus

$$
H=x_{0}+\overline{\bigcup_{0 \leq \lambda \leq d} \lambda \operatorname{conv} f(J \times H)}
$$

(cf. [6], [17]). As

$$
\mu(H) \leq d \mu(f(J \times H)) \leq k d \mu(H)<\mu(H),
$$

we conclude that $\mu(H)=0$ and consequently $H$ is weakly compact in $E$.

Let $\widetilde{H}=\{x \in C(J, E): x$ is Lipschitz-continuous with constant $M$ and $x(J) \subset H\}$. It is clear that $\widetilde{H}$ is a weakly compact subset of $C(J, E)$. Since

$$
F(x)(t)=x_{0}+\int_{0}^{t} f(s, x(s)) d s \in x_{0}+t \overline{\operatorname{conv}} f(J \times H) \subset H
$$

and

$$
\|F(x)(t)-F(x)(s)\| \leq\left|\int_{s}^{t}\|f(\tau, x(\tau))\| d \tau\right| \leq M|t-s|
$$

for $x \in \widetilde{H}$ and $t, s \in J$, it follows that $F: \widetilde{H} \rightarrow \widetilde{H}$.

Using the Lebesgue dominated convergence theorem for the Pettis integral, we deduce that $F$ is weakly-weakly sequentially continuous. But $\widetilde{H}$ is weakly compact, so $F$ is weakly continuous on $\widetilde{H}$. By the SchauderTikhonov fixed point theorem, $F$ has a fixed point. Thus $S \neq \emptyset$ and since $S \subset H$, by the Eberlein-Shmul'yan theorem we deduce that $S$ is weakly compact.

For any $\eta>0$ denote by $S_{\eta}$ the set of all functions $u: J \rightarrow E$ satisfying the following conditions:

(i) $u(0)=x_{0},\|u(t)-u(s)\| \leq M|t-s|$ for $t, s \in J$;

(ii) $\sup _{t \in J}\left\|u(t)-x_{0}-\int_{0}^{t} f(s, u(s)) d s\right\|<\eta$;

(iii) $u(t) \in x_{0}+\bigcup_{0 \leq \lambda \leq t} \lambda \overline{\operatorname{conv}} f(J \times H)=: H_{t}$.

The set $S_{\eta}$ is nonempty as $S \subset S_{\eta}$. 
Let $\beta=\min (d, \eta / M)$. For any $\varepsilon \in(0, \beta)$ we define a function $v(\cdot, \varepsilon)$ by

$$
v(t, \varepsilon)= \begin{cases}x_{0} & \text { for } 0 \leq t \leq \varepsilon, \\ x_{0}+\int_{0}^{t-\varepsilon} f(s, v(s, \varepsilon)) d s & \text { for } \varepsilon<t \leq d .\end{cases}
$$

We have $v(0, \varepsilon)=x_{0}$ and $\|v(t, \varepsilon)-v(s, \varepsilon)\| \leq M|t-s|$, so $v(\cdot, \varepsilon)$ satisfies (i). Furthermore,

$$
\begin{aligned}
& \left\|v(t, \varepsilon)-x_{0}-\int_{0}^{t} f(s, v(s, \varepsilon)) d s\right\| \\
& \quad=\left\{\begin{array}{ll}
\left\|\int_{0}^{t} f(s, v(\varepsilon, s)) d s\right\| & \text { for } 0 \leq t \leq \varepsilon \\
\left\|\int_{t-\varepsilon}^{t} f(s, v(\varepsilon, s)) d s\right\| & \text { for } \varepsilon \leq t \leq d
\end{array}\right\} \leq M \varepsilon<\eta,
\end{aligned}
$$

and $v(\cdot, \varepsilon)$ satisfies (ii).

We now prove that $v(\cdot, \varepsilon)$ satisfies (iii). If $0 \leq t \leq \varepsilon$, then $v(t, \varepsilon)=$ $x_{0} \in H_{t}$. Let $\varepsilon<t \leq 2 \varepsilon$. Then by the mean value theorem for the Pettis integral,

$$
v(t, \varepsilon)=x_{0}+\int_{0}^{t-\varepsilon} f\left(s, x_{0}\right) d s \in x_{0}+(t-\varepsilon) \overline{\operatorname{conv}} f(J \times H) \subset H_{t} \subset H .
$$

We extend the above procedure to the interval $(2 \varepsilon, 3 \varepsilon]$ and we find that $v(t, \varepsilon) \in H_{t} \subset H$. By induction, we obtain our assertion, and so $v(\cdot, \varepsilon) \in$ $S_{\eta} \subset \widetilde{H}$.

Now, we prove the connectedness of $S_{\eta}$. Put $v(\cdot, \varepsilon)=v_{\varepsilon}$. Then

$$
v_{\varepsilon}(t)= \begin{cases}x_{0}, & 0 \leq t \leq \varepsilon, \\ F\left(v_{\varepsilon}\right)(t-\varepsilon), & \varepsilon<t \leq d .\end{cases}
$$

We show that the mapping $\varepsilon \rightarrow v_{\varepsilon}(\cdot)$ is weakly continuous from $(0, \beta)$ into $C(J, E)$.

Let $0<\varepsilon<\delta \leq d$. For $t \in[0, \varepsilon]$,

$$
\left|x^{*}\left(v_{\varepsilon}(t)-v_{\delta}(t)\right)\right|=0 .
$$

For $t \in(\varepsilon, \delta]$,

$$
\begin{aligned}
\left|x^{*}\left(v_{\varepsilon}(t)-v_{\delta}(t)\right)\right| & =\left|x^{*} \int_{0}^{t-\varepsilon} f\left(s, v_{\varepsilon}(s)\right) d s\right| \\
& \leq\left\|x^{*}\right\| M|t-\varepsilon| \leq\left\|x^{*}\right\| M|\delta-\varepsilon| .
\end{aligned}
$$


Now, for $t \in(\delta, 2 \delta]$ we have

(3) $\left|x^{*}\left(v_{\varepsilon}(t)-v_{\delta}(t)\right)\right|$

$$
\begin{aligned}
= & \left|x^{*}\left(\int_{0}^{t-\varepsilon} f\left(s, v_{\varepsilon}(s)\right) d s-\int_{0}^{t-\delta} f\left(s, v_{\delta}(s)\right) d s\right)\right| \\
= & \left|x^{*}\left(F\left(v_{\varepsilon}\right)(t-\varepsilon)-F\left(v_{\delta}\right)(t-\delta)\right)\right| \\
\leq & \left|x^{*}\left(F\left(v_{\varepsilon}\right)(t-\varepsilon)-F\left(v_{\varepsilon}\right)(t-\delta)\right)\right| \\
& +\left|x^{*}\left(F\left(v_{\varepsilon}\right)(t-\delta)-F\left(v_{\delta}\right)(t-\delta)\right)\right| \\
\leq & \mid x^{*}\left(F\left(v_{\varepsilon}\right)(t-\delta)-F\left(v_{\delta}\right)(t-\delta)\right) \\
& +\| x^{*}|| M|t-\varepsilon-t+\delta| \\
= & \left|x^{*}\left(F\left(v_{\varepsilon}\right)(t-\delta)-F\left(v_{\delta}\right)(t-\delta)\right)\right|+\left\|x^{*}\right\| M|\delta-\varepsilon| .
\end{aligned}
$$

But $F$ is weakly continuous and since $t-\delta \leq \delta$, by using (2) we conclude that the map $\varepsilon \rightarrow v_{\varepsilon}(t)$ from $(0, \beta)$ into $E$ is weakly continuous for each $t \in[0,2 \delta]$. By induction, we can repeat this argument to deduce that the map $\varepsilon \rightarrow v_{\varepsilon}(\cdot)$ from $(0, \beta)$ into $(C(J, E), w)$ is continuous (cf. [13], Lemma 1.9). Therefore the set $V:=\left\{v_{\varepsilon}(\cdot): 0<\varepsilon<\beta\right\}$ is connected in $(C(J, E), w)$.

Let $x \in S_{\eta}$. Choose $\varepsilon>0$ such that $0<\varepsilon<\beta$ and

$$
\sup _{t \in J}\left\|x(t)-x_{0}-\int_{0}^{t} f(s, x(s)) d s\right\|+M \varepsilon<\eta .
$$

For any $p, 0 \leq p \leq d$, we define a function $y(\cdot, p)$ by the formula

$$
y(t, p)= \begin{cases}x(t) & \text { for } 0 \leq t \leq p, \\ x(p) & \text { for } p<t \leq \min (d, p+\varepsilon], \\ x(p)+\int_{p}^{t-\varepsilon} f(s, y(s, p)) d s & \text { for } \min (d, p+\varepsilon) \leq t \leq d .\end{cases}
$$

By repeating the above considerations for $y(\cdot, p)$ instead of $v(\cdot, \varepsilon)$ we establish that $y(\cdot, p) \in S_{\eta}$ for each $p \in[0, d]$ and we prove the continuity of the mapping $p \rightarrow y(\cdot, p)$ from $J$ into $(C(J, E), w)$ (for more details see [11] and $[18])$.

Consequently, the set

$$
T_{x}=\{y(\cdot, p): 0 \leq p \leq d\}
$$

is connected in $(C(J, E), w)$. As $y(\cdot, 0)=v(\cdot, \varepsilon) \in V \cap T_{x}$, the set $V \cap T_{x}$ is also connected, and the same is true of

$$
W=\bigcup_{x \in S_{\eta}} T_{x} \cup V .
$$


Moreover, $S_{\eta} \subset W$, because $x=y(\cdot, d) \in T_{x}$ for each $x \in S_{\eta}$. On the other hand, $W \subset S_{\eta}$, since $T_{x} \subset S_{\eta}$ and $V \subset S_{\eta}$. Finally, $S_{\eta}=W$ is a connected subset of $(C(J, E), w)$.

Denote by $\bar{S}_{\eta}$ the closure of $S_{\eta}$ in $(C(J, E), w)$. Obviously, for each $u \in \bar{S}_{\eta}$ and $t, s \in J$,

$$
u(0)=x_{0}, \quad\|u(t)-u(s)\| \leq M|t-s|
$$

and $u(t) \in H_{t}$ because $H_{t}$ is closed convex.

We now show that

$$
\left\|u(t)-x_{0}-\int_{0}^{t} f(s, u(s)) d s\right\| \leq \eta \quad \text { for } u \in \bar{S}_{\eta} \text { and } t \in J .
$$

If $\bar{u} \in \bar{S}_{\eta}$, then for any $\varepsilon>0$ and $y^{*} \in C(J, E)^{*}$, there exists $u \in S_{\eta}$ such that $\left|y^{*}(u-\bar{u})\right|<\varepsilon$ and $\left|y^{*}(F u-F \bar{u})\right|<\varepsilon$ as $F$ is weakly continuous. Let $y^{*}$ be such that $\left\|y^{*}\right\|=1$ and $\left|y^{*}(\bar{u}-F \bar{u})\right|=\|\bar{u}-F \bar{u}\|$. Then

$$
\begin{aligned}
\|\bar{u}-F \bar{u}\| & =\mid y^{*}(\bar{u}-F(\bar{u}) \mid \\
& \leq\left|y^{*}(\bar{u}-u)\right|+\left|y^{*}(u-F u)\right|+\left|y^{*}(F u-F \bar{u})\right|<\varepsilon+\eta+\varepsilon .
\end{aligned}
$$

Since the last inequality is satisfied for each $\varepsilon>0$ we get (5).

We see that $\bar{S}_{\eta}$ is a weakly closed strongly equicontinuous subset of $C(J, E)$ such that $u(t) \in H$ for each $u \in \bar{S}_{\eta}$ and $t \in J$. As $H$ is weakly compact, $\bar{S}_{\eta}$ is compact in $(C(J, E), w)$.

Put $V_{n}=\bar{S}_{1 / n}$ for $n=1,2, \ldots$ Then $V_{n}$ is a decreasing sequence of nonempty compact connected subsets of $(C(J, E), w)$, and therefore the intersection $S=\bigcap_{n=1}^{\infty} V_{1 / n}$ is nonempty, compact and connected in $(C(J, E), w)$. From (5) it follows that $S$ is the set of all solutions of (1) defined on $J$, which completes the proof.

It is not difficult to prove that if $f(\cdot, x)$ is scalarly measurable, $f(t, \cdot)$ is weakly-weakly continuous, $f$ is bounded, and $E$ is a WCG Banach space ([8]), then for each absolutely strongly continuous function $x(\cdot), f(\cdot, x(\cdot))$ is Pettis-integrable (cf. [8], p. 99).

Note that our pseudo-solution is pseudo-differentiable, and may be nowhere weakly differentiable (see [4], Example 3, or [15]). It is well known that the integral of a weakly continuous function is weakly differentiable with respect to the right endpoint of the integration interval and its derivative equals the integrand at that point (see [13], Lemma 2.3, [16]). In this case a pseudo-solution is, in fact, a weak solution. Therefore as special cases we obtain the following corollaries.

COROllary 1 ([11]). Let $E$ be a weakly sequentially complete Banach space. Assume that $f$ is weakly-weakly continuous on $J \times B_{r}$. In addition, let $f$ satisfy $(*)$. Then the set $S$ of all weak solutions of the Cauchy problem (1) on $J$ is a continuum in $C_{\mathrm{w}}(J, E)$. 
Corollary 2 ([18]). If $E$ is a reflexive Banach space, and $f$ is weaklyweakly continuous, then the set $S$ of all weak solutions of the Cauchy problem (1) on $J$ is a continuum in $C_{\mathrm{w}}(J, E)$.

Now, as a corollary we can obtain the result on existence of strong $C$-solutions (cf. [4], Corollary 3, [1], [10]).

Proposition 1. Suppose that $f$ satisfies the following assumptions:

(i) for each $t \in J, f(t, \cdot)$ is weakly-weakly continuous,

(ii) for each $x \in B, f(\cdot, x)$ is strongly measurable on $J$,

(iii) $f$ satisfies $(*)$.

Then all pseudo-solutions of the problem (1) are, in fact, strong $C$-solutions, and so the set $S$ of all strong $C$-solutions is a continuum in $(C(J, E), w)$.

This is a simple consequence of our Theorem 1 and Corollary 3 from [4] (cf. also [10], Corollary 3).

Observe that if the space $E$ is separable then each pseudo-solution of (1) is a strong Carathéodory solution (see [1]).

\section{References}

[1] O. Arino, S. Gautier and J. P. P e not, A fixed point theorem for sequentially continuous mappings with application to ordinary differential equations, Funkc. Ekvac. 27 (1984), 273-279.

[2] J. B a n á and K. Go e bel, Measures of Noncompactness in Banach Spaces, Lecture Notes in Pure and Appl. Math. 60, Marcel Dekker, New York, 1980.

[3] J. B a n aś and J. R ive ro, On measure of weak noncompactness, Ann. Mat. Pura Appl. 125 (1987), 213-224.

[4] M. Cichoń, Weak solutions of differential equations in Banach spaces, Discuss. Math. 15 (1994) (in press).

[5] E. Cramer, V. Lakshmikantham and A. R. Mitchell, On the existence of weak solutions of differential equations in nonreflexive Banach spaces, Nonlinear Anal. 2 (1978), 169-177.

[6] S. J. D a her, On a fixed point principle of Sadovskii, ibid., 643-645.

[7] F. S. De B lasi, On a property of the unit sphere in a Banach space, Bull. Math. Soc. Sci. Math. R. S. Roumanie 21 (1977), 259-262.

[8] J. Diestel and J. J. Uhl J r., Vector Measures, Math. Surveys 15, Amer. Math. Soc., Providence, R.I., 1977.

[9] A. F. Filippov, Differential Equations with Discontinuous Right-Hand Side, Nauka, Moscow, 1985 (in Russian).

[10] W. J. Knight, Solutions of differential equations in B-spaces, Duke Math. J. 41 (1974), 437-442.

[11] I. K u b i a c z y k, Kneser type theorems for ordinary differential equations in Banach spaces, J. Differential Equations 45 (1982), 139-146.

[12] I. K u bi a c z y k and S. S z ufla, Kneser's theorem for weak solutions of ordinary differential equations in Banach spaces, Publ. Inst. Math. (Beograd) 32 (1982), 99-103. 
[13] A. R. Mitchell and Ch. Smith, An existence theorem for weak solutions of differential equations in Banach spaces, in: Nonlinear Equations in Abstract Spaces, V. Lakshmikantham (ed.), 1978, 387-404.

[14] N. S. Papageorgiou, Kneser's theorems for differential equations in Banach spaces, Bull. Austral. Math. Soc. 33 (1986), 419-434.

[15] B. J. P ettis, On integration in vector spaces, Trans. Amer. Math. Soc. 44 (1938), 277-304.

[16] A. S z e p, Existence theorem for weak solutions of ordinary differential equations in reflexive Banach spaces, Studia Sci. Math. Hungar. 6 (1971), 197-203.

[17] S. S z u f l a, Some remarks on ordinary differential equations in Banach spaces, Bull. Acad. Polon. Sci. Math. 16 (1968), 795-800.

[18] —, Kneser's theorem for weak solutions of ordinary differential equations in reflexive Banach spaces, ibid. 26 (1978), 407-413.

FACULTY OF MATHEMATICS AND COMPUTER SCIENCE

ADAM MICKIEWICZ UNIVERSITY

MATEJKI $48 / 49$

60-769 POZNAŃ, POLAND

Reçu par la Rédaction le 20.6.1993 